\title{
Correction to: Effective Solar Indices for Ionospheric Modeling: A Review and a Proposal for a Real-Time Regional IRI
}

\author{
A. Pignalberi ${ }^{1} \cdot$ M. Pezzopane $^{2} \cdot$ R. Rizzi $^{1} \cdot$ I. Galkin $^{3}$
}

Published online: 23 November 2017

(C) Springer Science+Business Media B.V., part of Springer Nature 2017

\section{Correction to: Surv Geophys \\ https://doi.org/10.1007/s10712-017-9438-y}

In the original publication of the article, under Section 2.1, the sentence in the eighth paragraph that reads as "It has been demonstrated...very disturbed periods." should read as "It has been demonstrated (Mirò Amarante et al. 2007) that the ionosphere shows small scale variations that global or regional empirical models describe hardly, especially for very disturbed periods."

In the eleventh paragraph under Section 3.2, the sentence that reads as "In formulas (3)-(4),...respectively." should read as "In formulas (3)-(4), $U_{2 j, k}^{\text {low }}$ and $U_{2 j, k}^{\text {high }}$ (similarly for $2 j-1$ terms) are the coefficient values calculated for $I G_{12}$ or $R_{12}$ equal to 0 (low solar activity) and equal to 100 (high solar activity), respectively."

Finally, under Section 3.3.2, the sentence in the third paragraph that reads as "In this case, the... or small values of $h$." should read as "In this case, the experimental variogram has a parabolic behavior near the origin, that is, it is proportional to $h^{2}$ for small values of $h . "$

The original article can be found online at https://doi.org/10.1007/s10712-017-9438-y.

\section{A. Pignalberi}

alessio.pignalberi2@unibo.it

1 Dipartimento di Fisica e Astronomia, Università di Bologna “Alma Mater Studiorum”, Viale Berti Pichat 6/2, 40127 Bologna, Italy

2 Istituto Nazionale di Geofisica e Vulcanologia, Via di Vigna Murata 605, 00143 Rome, Italy

3 Space Science Laboratory, University of Massachusetts, 600 Suffolk Street, Lowell, MA 01854, USA 